\title{
Influence of the Magnesium Treatment in a Georg Fischer Converter Mathias Lueben
}

\author{
Georg Fischer Automobilguss GmbH, Julius-Bührer-Str. 12, 78224 Singen, Germany \\ mathias.lueben@georgfischer.com
}

Keywords: thermal analysis, magnesium treatment, Georg Fischer converter, ductile iron, pseudorecalescence

\begin{abstract}
With up-to-date thermal analysis equipment, it is possible to control and regulate the melting and treatment process of ductile iron, more or less at every step of the process. Changes in the composition can be handled by controlling and then regulating each single step.

The complete process, starting with the melting in a cupola furnace, followed by the holding process in an induction channel furnace and finally the magnesium treatment in a converter has been analysed. A correlation to control and regulate the eutectic undercooling of the melt has been developed.
\end{abstract}

\section{Introduction}

Is there any possibility to influence the final nucleation properties of the melt, after the $\mathrm{Mg}$ treatment in a converter by changing parameters before treatment?

The general opinion of the Mg-treatment converter-process with addition of pure magnesium for producing ductile iron is, that there is nothing, regarding the nucleation, that survives the powerful reaction, because the iron is completely white solidified. The final nucleation properties of the iron are basically damaged, which is generally accepted. This result is more positively expressed a "homogeneous metallurgical state". [1] Accordingly, the base iron properties cannot be of any influence to the final nucleation properties.

But the practical experience as well as the latest research results show that there has to be some connection.

\section{Experimental Methods and Results}

It is possible to influence the final nucleation properties directly by controlling the melting and holding process. Furthermore, it is possible to calculate the eutectic undercooling respectively the lower eutectic temperature after magnesium treatment, using parameters measured by thermal analysis before magnesium treatment under certain conditions.

The continuously used test piece for this research is the AccuVo® double chamber cup, see figure 1. It is a thermal analysis cup with two comparable chambers, one chamber is plain, without any addition and the other chamber can be charged with, for example an inoculant to check in one measurement, direct comparable the nucleation conditions. 


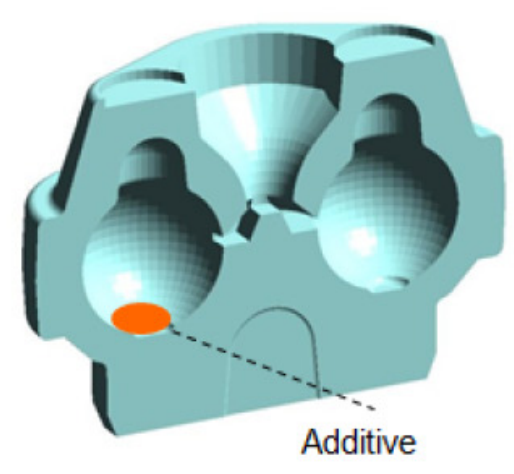

a) Empty Cup

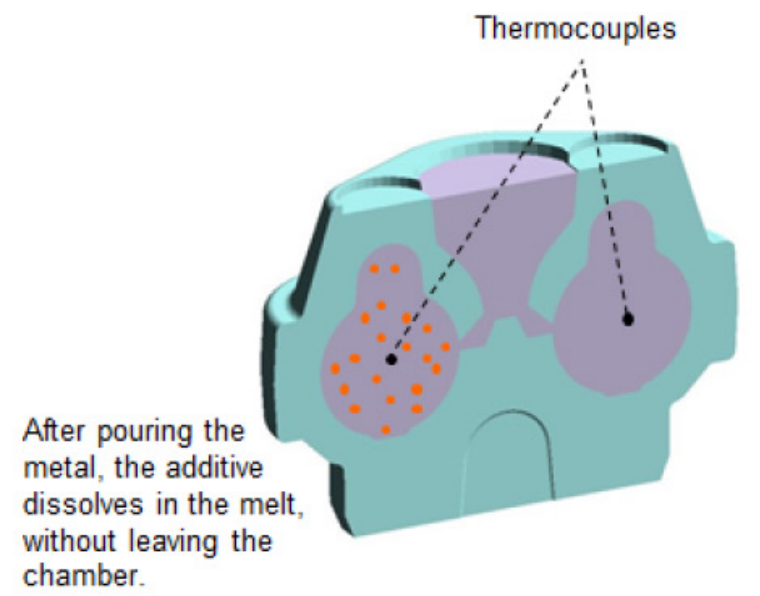

b) Cup after filling

Figure 1: A drawing of the AccuVo® double chamber cup, cut vertically into two halves. [2]

a) The empty cup with an additive in the left chamber, for example inoculant.

b) The cup after filling with melt, marked the positions of the thermocouples.

Figure 2 shows a typical cooling curve of a hypoeutectic melt, treated with pure magnesium in a converter, measured in an AccuVo ${ }^{\circledR}$ double chamber cup. The blue coloured curve shows the uninoculated, metastable solidified cooling and the orange curve shows the inoculated, stable solidified cooling. In figure 2 the main characteristic points are labelled.

To understand the connection of the different steps of the production process different trials with some remarkable results have been conducted. At the beginning of production, at the cupola furnace, there is a lamellar graphite base iron, for production of ductile iron. The iron is melted with an output of up to 100 tons per hour and then stored in three induction channel furnaces with a total capacity of about 345 tons. The Mg-treatment is conducted in the converter with addition of pure Magnesium. For reference 10 tons of cast iron are treated with about $14 \mathrm{~kg}$ of pure Magnesium. 


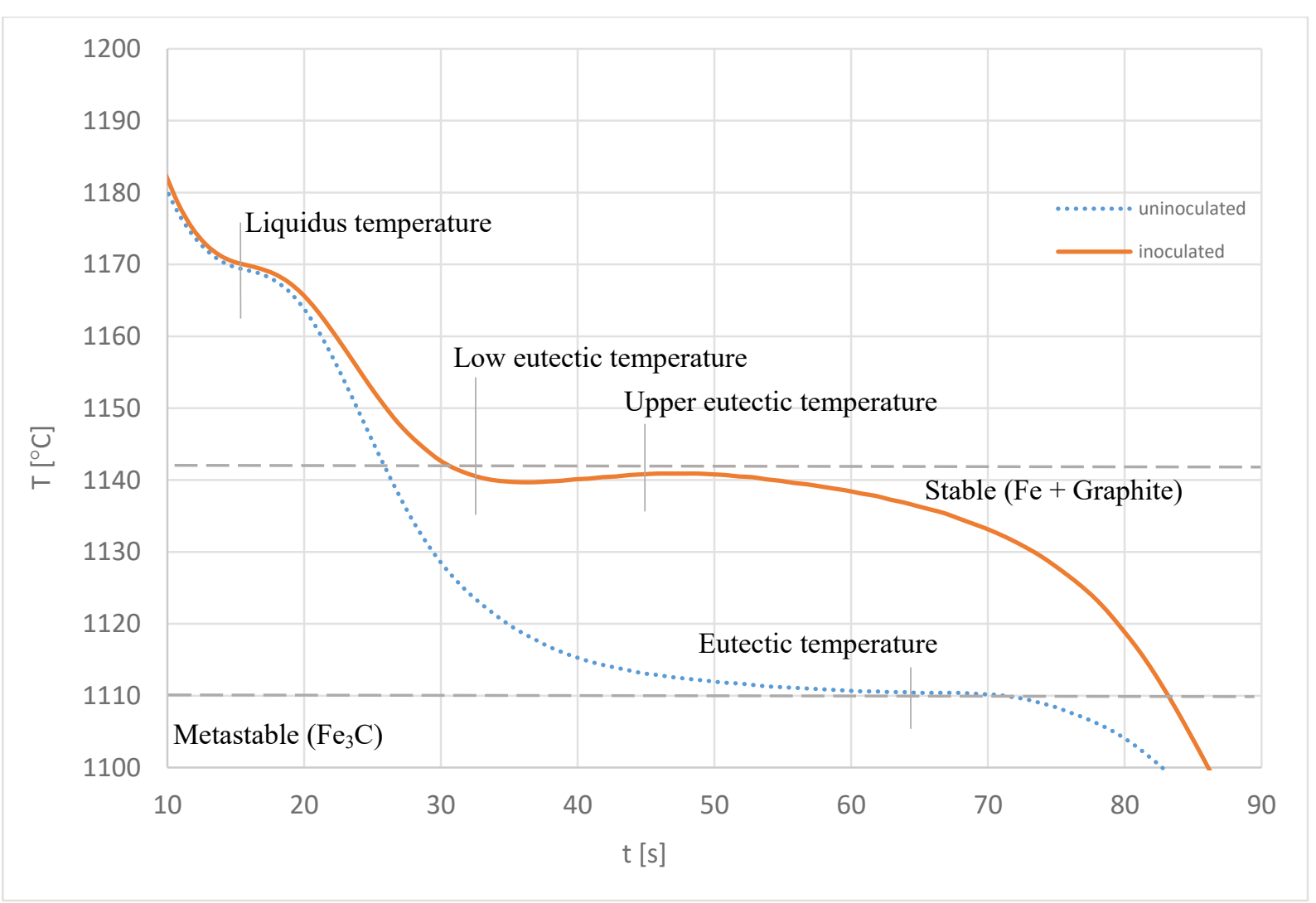

Figure 2: A typical cooling curve of a hypoeutectic melt, treated with pure magnesium in a converter, measured in an AccuVo ${ }^{\circledR}$ double chamber cup. With the uninoculated, metastable solidified cooling curve in blue and the inoculated, stable solidified cooling curve in orange. It also shows the main characteristic points.

Figure 3 shows the microstructure of a sample of the same melt before and after magnesium treatment. According to literature there is a relatively good correlation between the number of eutectic cells and the nodule count. The more the number of eutectic cells, the higher the nodule count. [3] [4]

Based on this research it is possible to get a defect-free microstructure under economic aspects, by regulating the melting process in the desired way.

The first task is the physical result. How does the microstructure of the cast part depend on the condition of the treated melt? Figure 4 shows, that the nodule count in the Mg-treated iron rises with a decrease of the eutectic undercooling in inoculated condition with in this case $\mathrm{R}^{2}=0.63$.

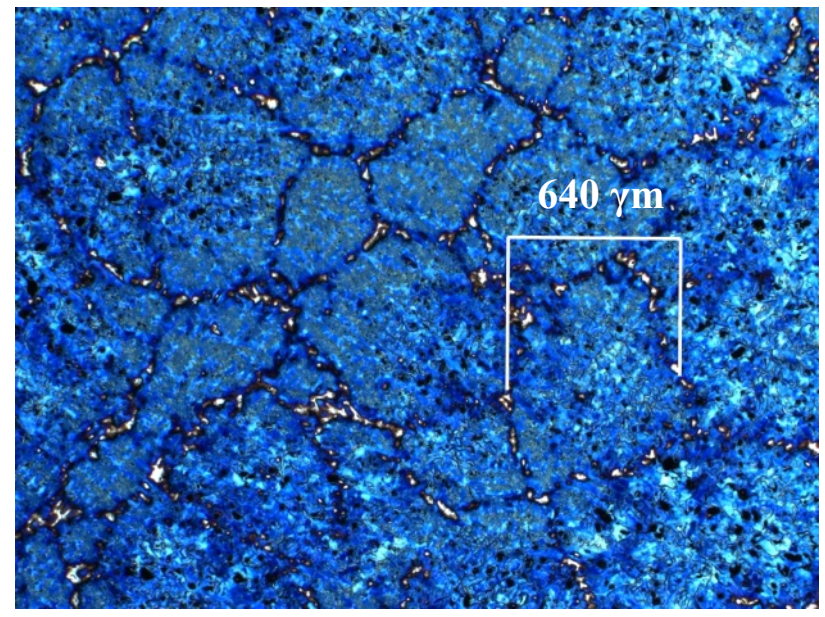

Figure 3:

Top: Polished section of the thermal analysis sample before magnesium treatment (scaling factor 2,58 $\mathrm{\gamma m} / \mathrm{pixel}$ ), etched for eutectic cells. 


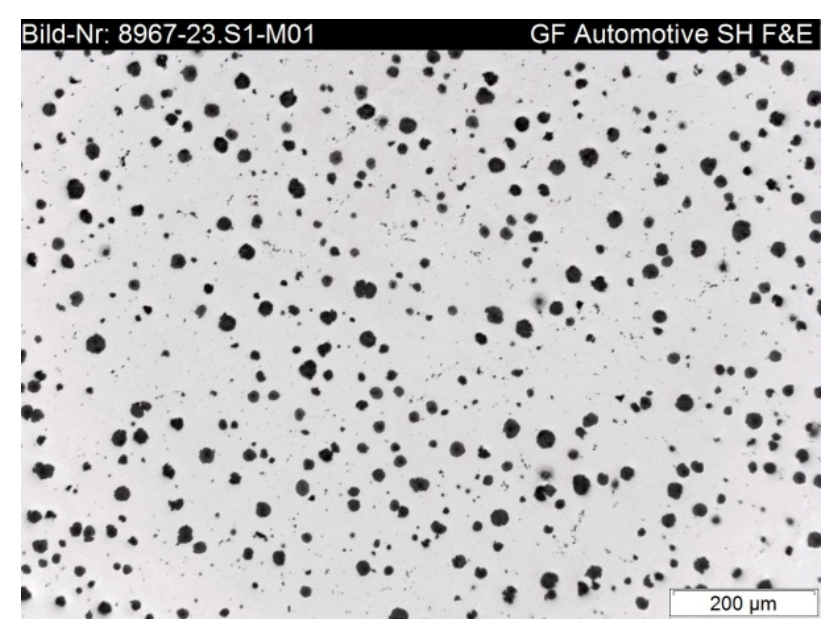

Bottom: Polished section of the thermal analysis sample after magnesium treatment.

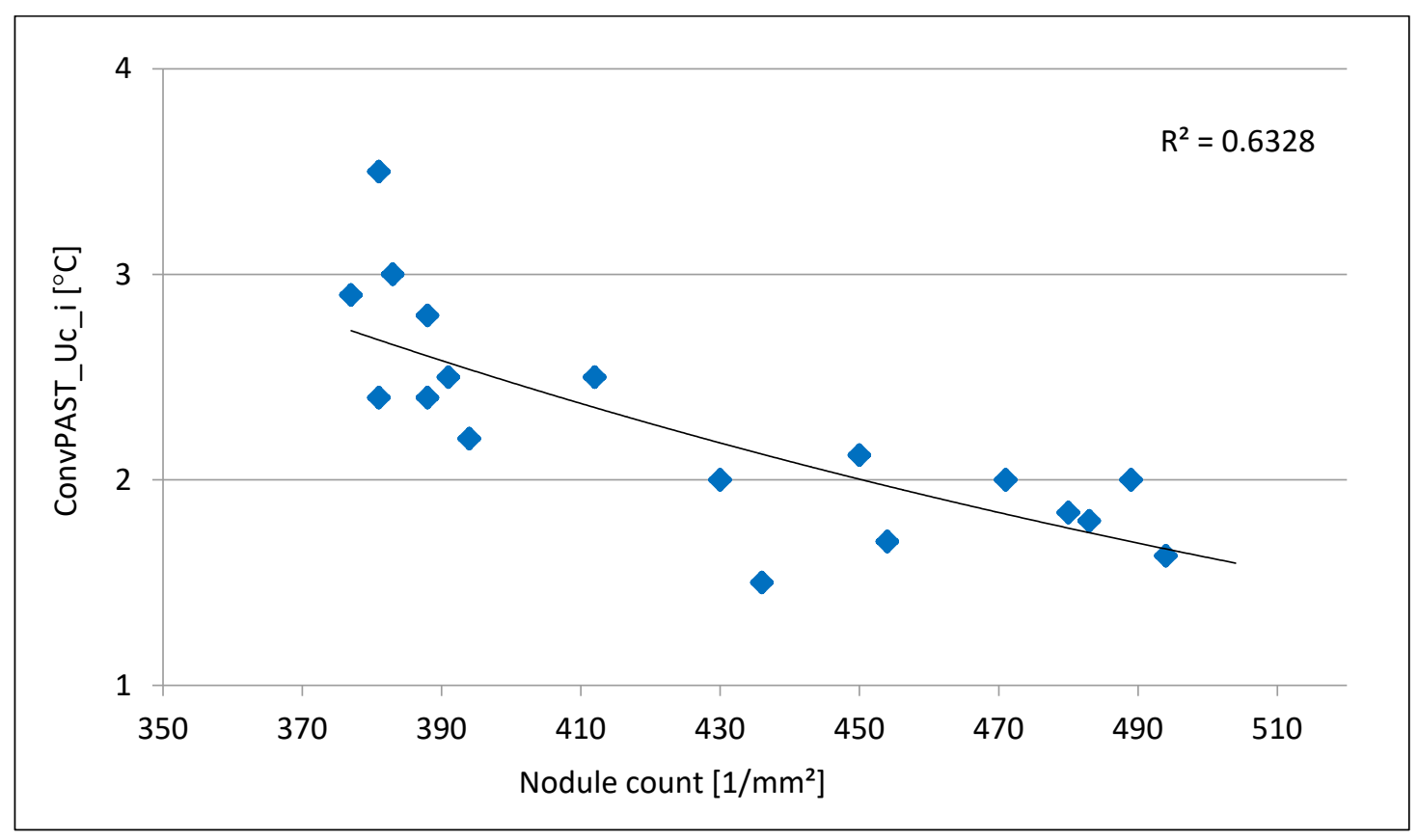

Figure 4: Graph shows the increasing nodule count with decreasing eutectic undercooling. The term ConvPAST_Uc_i is defined as the eutectic undercooling after Mg-treatment and inoculation.

Further trials show that it is possible to approximate the eutectic undercooling after inoculation, see equation 1. This correlation for example has been developed out of a data set with 170 rows and 74 columns, done with a computer program for multiple regression analyses. The variables a, b and $\mathrm{c}$ have to be determined with the specific parameters of the particular process and additional to that in dependence to varying process situations.

$$
\text { ConvPast_Uc_i=a+b*Conv } \operatorname{Pr} e_{-} E u t R C_{-} u+c * C o n v \operatorname{Pr} e_{-} U c_{-} u
$$

The following shortcuts have been used for equation 1:

- ConvPast_Uc_i: eutectic undercooling after Mg-treatment and inoculation

- ConvPre_EutRC_u: eutectic recalescence, without inoculant and before Mg-treatment

- ConvPre_Uc_u: eutectic undercooling, without inoculant and before Mg-treatment.

Figure 5 shows the dependence of the by figure 1 calculated and also measured values for the analysed process with $\mathrm{R}^{2}=0.74$. The direct correlation is obvious. 


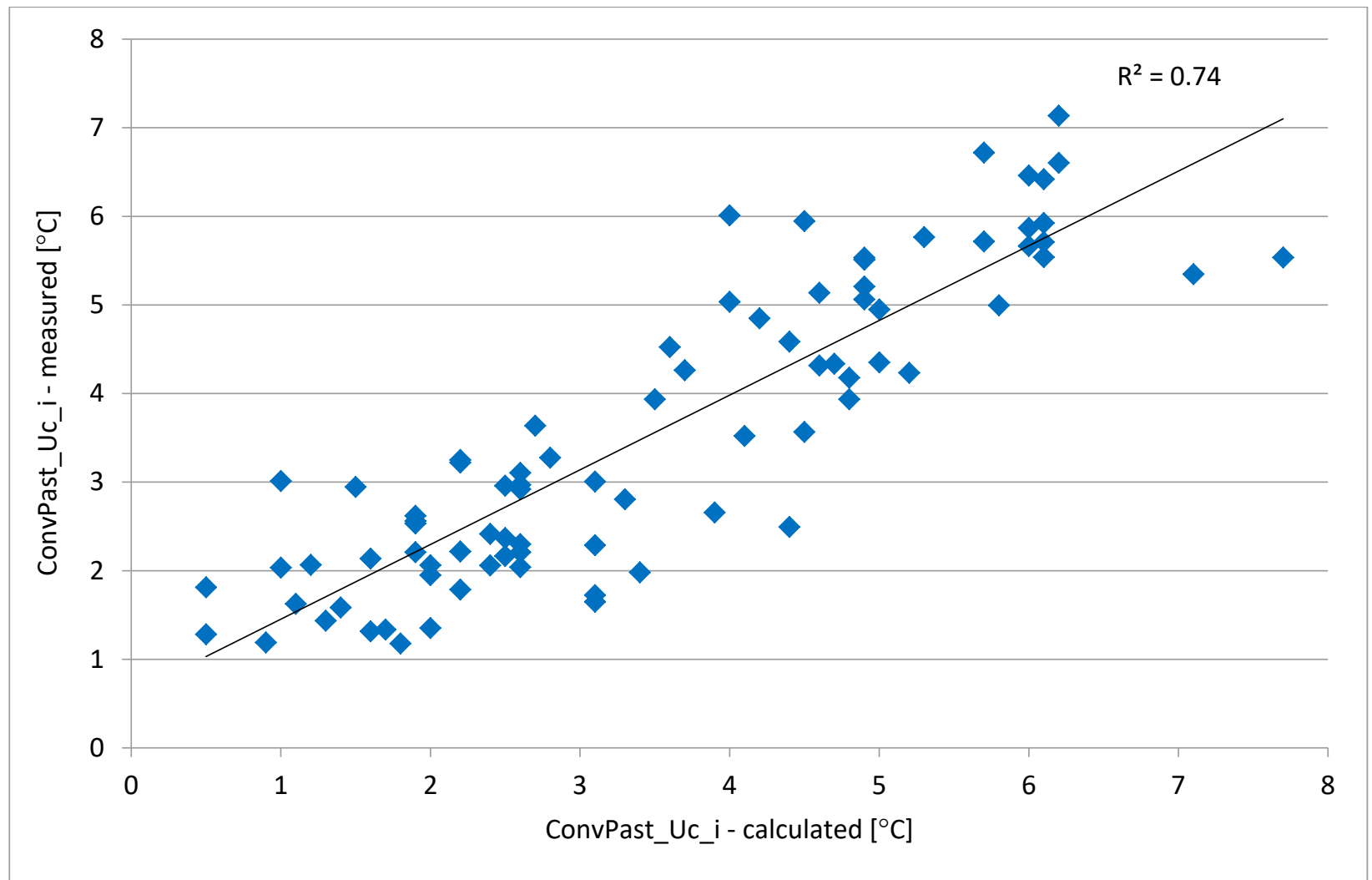

Figure 5: The eutectic undercooling after Mg-treatment and inoculation, comparison between calculation by figure 1 and measurement.

Figure 6 shows, that the nodule count respectively the particle density per $\mathrm{mm}^{2}$ in the $\mathrm{Mg}$-treated iron rises with an increase of the lower eutectic temperature in inoculated condition with in this case $\mathrm{R}^{2}=0.90$.

Further trials show that it is possible to approximate the eutectic undercooling after inoculation.

\section{Discussion}

The completion of the process could be a dynamic controlled addition of inoculant. In dependence to the thermal analysis result, more precisely the eutectic undercooling respectively the lower eutectic temperature the amount of added inoculant regulates the particle density in the cast part, see figure 6 . 


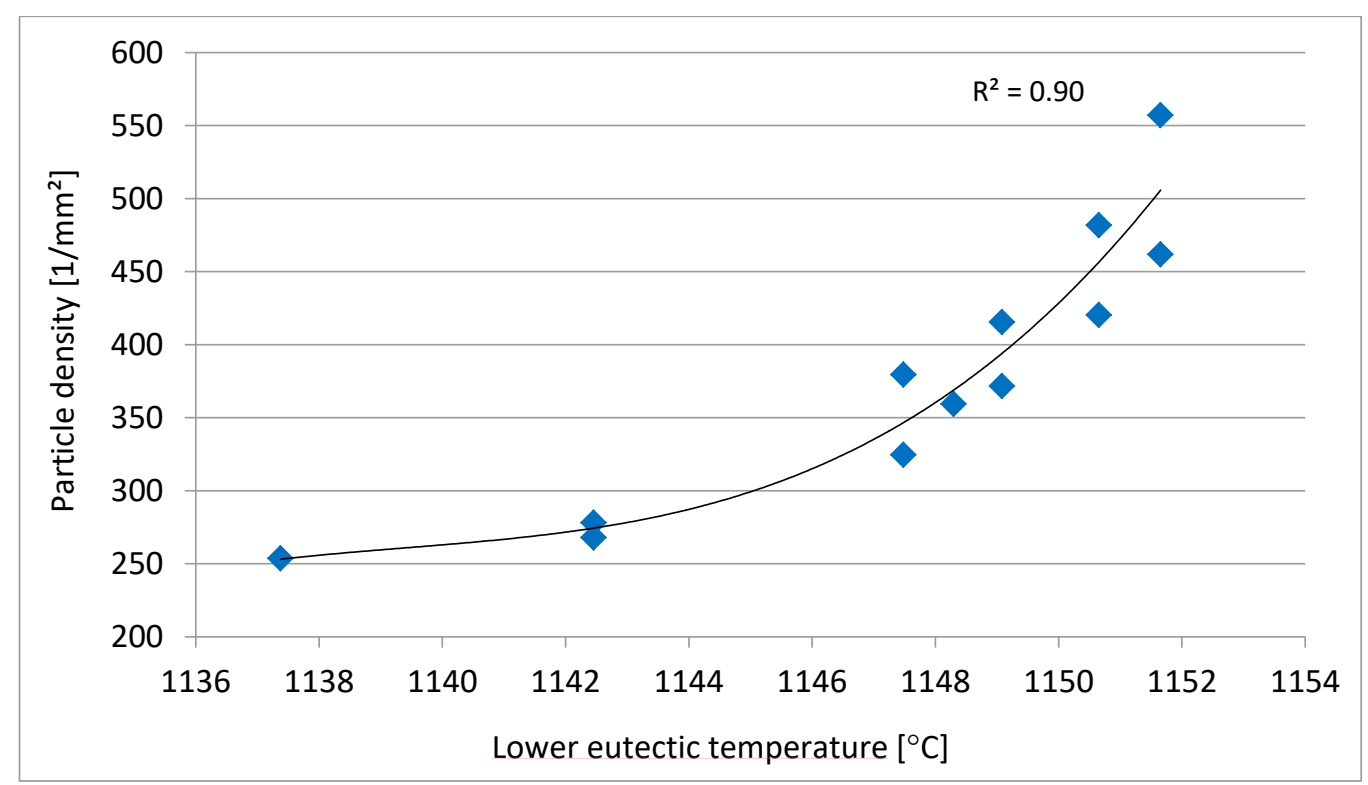

Figure 6: Particle density versus lower eutectic temperature.

Another remarkable parameter is the, so called pseudo-recalescence of magnesium treated iron in the metastable cooling curve, after the lower eutectic temperature. The pseudo-recalescence is depicted in figure 7 (point 1), with the associated polished section in figure 8 . There occurs a formation of a graphite phase because of the outstanding nucleation conditions of the base melt. The mechanism of this formation is currently not completely explainable. Further trials are necessary to understand, control and use this kind of graphite formation.

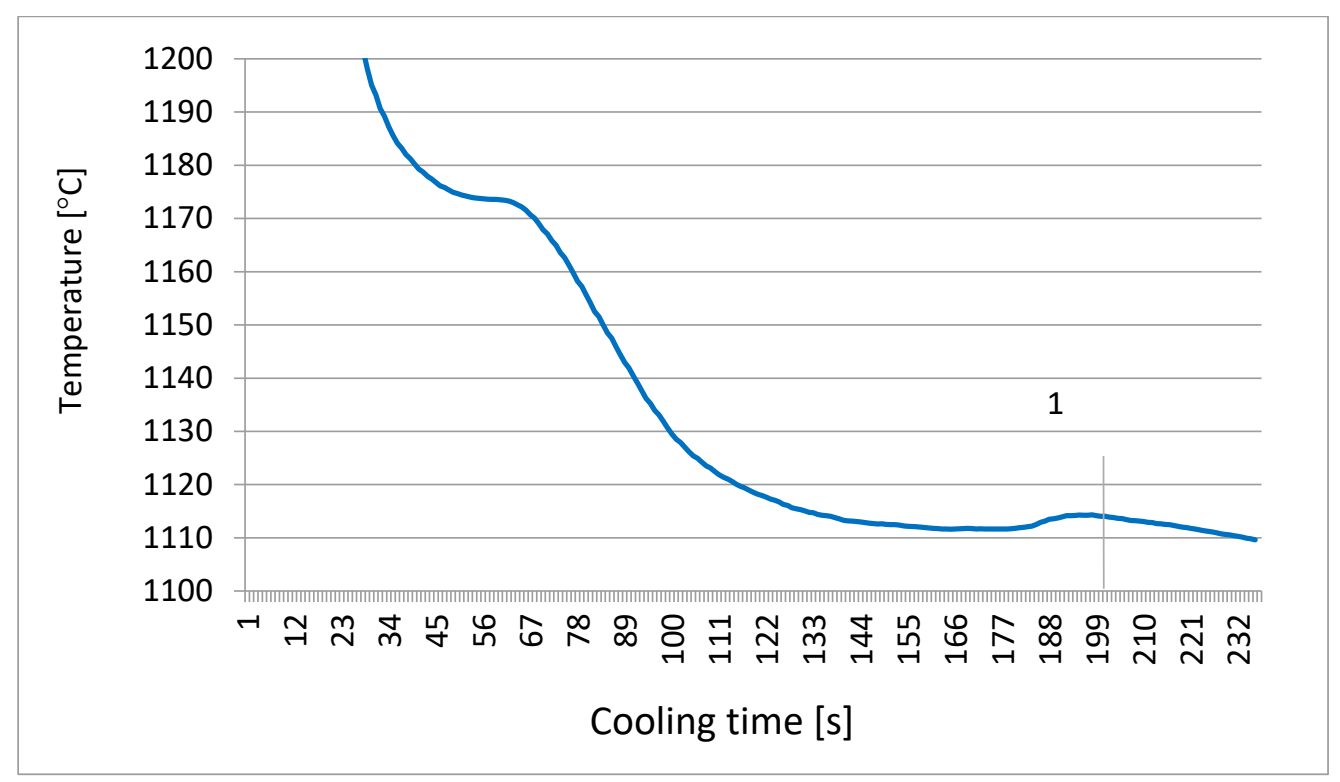

Figure 7: Metastable cooling curve of magnesium treated iron with a eutectic recalescence of $2.7^{\circ} \mathrm{C}$ (point 1) after the lower eutectic temperature. 


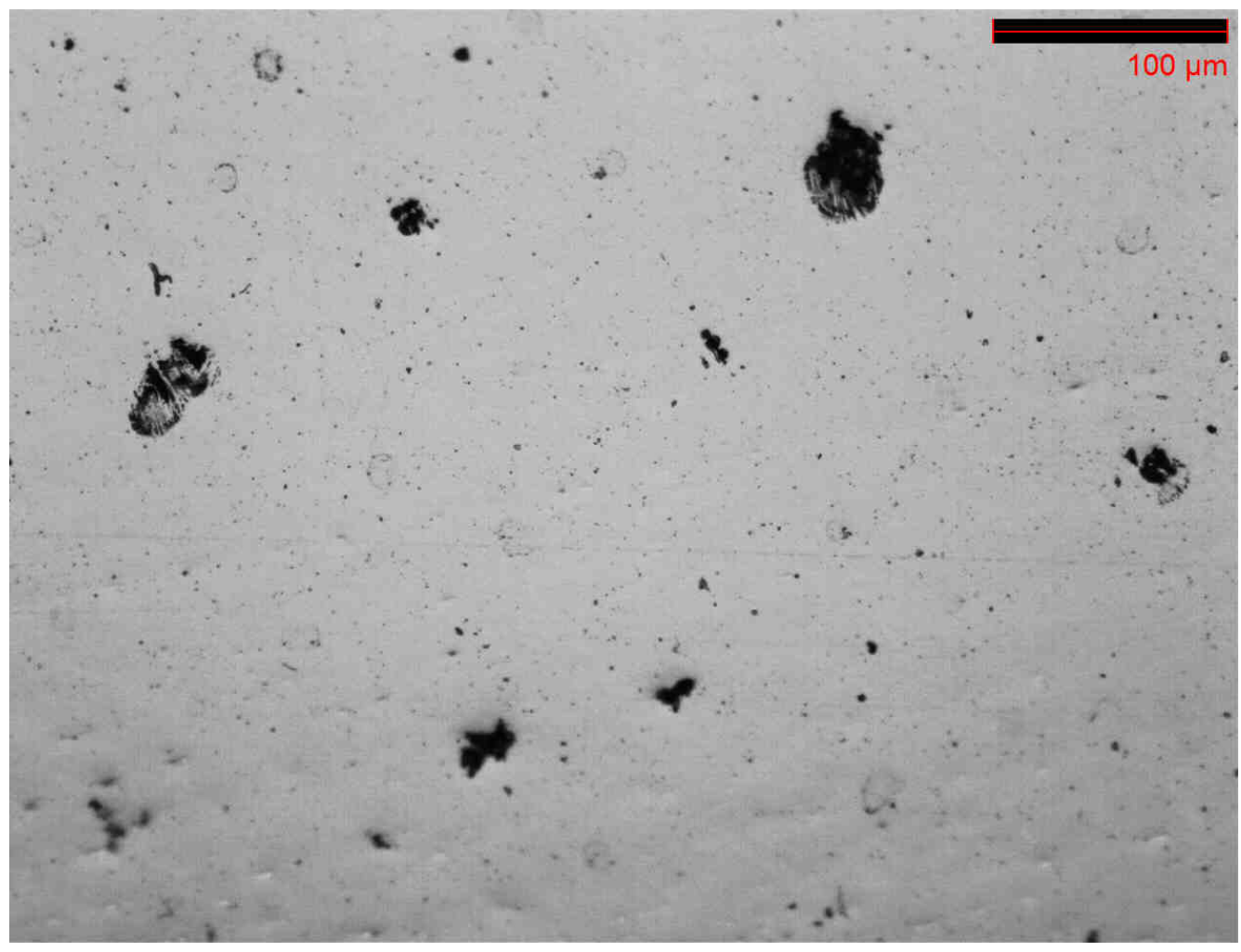

Figure 8: Graphite phase in a magnesium treated, metastable solidified cast iron.

\section{Conclusion}

As stated above it is possible to regulate the result of your melting process when using a converter for Mg-treatment to produce ductile iron by changing parameters of the melting process. A change, for example in the charge make up does have an influence on the final nucleation properties.

Using this knowledge, you are able to react directly to changes in the composition of the ingredients under economic aspects.

\section{Acknowledgements}

I would like to thank Georg Fischer Automotive, especially Robert Greibig, Dirk Lindemann, Werner Menk, Ulrich Stark and Jörg Winsberg.

\section{References}

[1] Wolfgang Knothe, Metallurgical Aspects of Production of Ductile Iron, $13^{\text {th }}$ International Licensee Conference, 1997.

[2] Vitor Anjos, Use of Thermal Analysis to Control the Solidification Morphology of Nodular Cast Irons and Reduce Feeding Needs, doctoral thesis, Duisburg, 2015.

[3] Klaus Röhrig, Legiertes Gusseisen, published in Giesserei-Praxis, Erkrath, 2012.

[4] Impfen von Gusseisen, Elkem AS, 2012. 